Research Paper

\title{
Microarray Analysis of Difierential Gene Expression Profile in Peripheral Blood Cells of Patients with Human Essential Hypertension
}

\author{
Melvin T. Korkor ${ }^{1}$, Fan Bo Meng ${ }^{1}$, Shen Yang Xing ${ }^{2}$, Mu Chun Zhang ${ }^{3}$, Jin Rui Guo1, Xiao Xue Zhu¹, Ping \\ Yang $^{1 \otimes}$
}

1. Department of Internal Medicine and Cardiology, China-Japan Union Hospital, Norman Bethune College of Medicine, Jilin University, China,

2. College of Animal Science and veterinary medicine, Jilin University, China

3. Department of Urology, China-Japan Union Hospital, Norman Bethune College of Medicine, Jilin University, China

$\triangle$ Corresponding author: Ping Yang, Ph.D, Department of Internal Medicine and Cardiology, China-Japan Union Hospital, Norman Bethune College of Medicine, Jilin University, China. Tel: 0086-431-84995091; Fax: 0086-431-84995091; E-mail: pyang@jlu.edu.cn

(c) Ivyspring International Publisher. This is an open-access article distributed under the terms of the Creative Commons License (http://creativecommons.org/ licenses/by-nc-nd/3.0/). Reproduction is permitted for personal, noncommercial use, provided that the article is in whole, unmodified, and properly cited.

Received: 2010.10.31; Accepted: 2011.02.21; Published: 2011.02.27

\begin{abstract}
The polygenic nature of essential hypertension and its dependence on environmental factors pose a challenge for biomedical research. We hypothesized that the analysis of gene expression profiles from peripheral blood cells would distinguish patients with hypertension from normotensives. In order to test this, total RNA from peripheral blood cells was isolated. RNA was reversed-transcribed and labeled and gene expression analyzed using significance Analysis Microarrays (Stanford University, CA, USA). Briefly, Significance Analysis Microarrays (SAM) thresholding identified 3I up-regulated and 18 down-regulated genes with fold changes of $\geq 2$ or $\leq 0.5$ and q-value $\leq 5 \%$ in expression. Statistically significantly gene ontology (GO) function and biological process differentially expressed in essential hypertension were MHC class II receptor activity and immune response respectively. Biological pathway analysis identified several related pathways which are associated with immune/inflammatory responses. Quantitative Real- Time RT-PCR results were consistent with the microarray results. The levels of $C$ - reactive protein were higher in hypertensive patients than normotensives and inflammation-related genes were increased as well. In conclusion, genes enriched for "immune/inflammatory responses" may be associated with essential hypertension. In addition, there is a correlation between systemic inflammation and hypertension. It is anticipated that these findings may provide accurate and efficient strategies for prevention, diagnosis and control of this disorder.
\end{abstract}

Key words: gene expression; hypertension, essential; inflammation; peripheral blood cells

\section{INTRODUCTION}

It has become fairly obvious that hypertension is an escalating clinical and public health problem for both developed and developing countries. According to recent report, as of the year 2000, 972 million people were afflicted worldwide and this figure is believed to rise to more than 1.56 billion by the year $2025(1,2)$.
Human essential hypertension enhances the risk of a variety of adverse sequelae, including stroke, heart failure, coronary heart disease and renal failure (3). Essential hypertension has been recognized as a complex, multifactorial, polygenic trait which involves multiple modulating genes and environmental 
factors. To all appearances, identifying genes involved in polygenic trait is much more difficult than monogenic trait. This is because of the weak relationship between allelic variation of individual loci and phenotypic expression of the trait.

Interestingly, with the advent of molecular genetics, substantial progress has been made in the last decade towards detection of genes involved in essential hypertension using two principal techniques: linkage analysis and exploration of candidate genes. Unfortunately, these traditional techniques are inevitably limited in that they normally allow researchers to study barely one or a few genes simultaneously. However, recent advances in molecular biology and technology have made it feasible to quantify the expression levels of thousands of genes simultaneously by utilizing the microarray technology.

Microarray technology is a robust high-throughput method use for efficient and accurate simultaneous expression measurement of thousands of genes $(4,5,6,7)$. The use of microarray technology for gene expression profiling has been successful and increasingly popular particularly in genetics and cancer research.

There is an extensive body of clinical and experimental evidence that inflammatory processes are important participants in both the initiation and development of hypertension (8). An evidence to this report is the elevated levels of proinflammatory markers such as Tumor necrosis factor- $\alpha$ (TNF- $\alpha$ ), Interleukin-6 (IL-6) and C-reactive protein observed in individuals with hypertensive (9). Blood leukocytes are the predominant cells that provide immunity, mediate stress and inflammation, and generate cytokines, chemokines and growth factors that exepend important pathological and physiological actions on peripheral tissues. In that event, dissecting peripheral blood cells gene expression patterns of individuals with hypertension could reflect the potential markers of the disease, including inflammatory response which is an important causal pathway leading to hypertension. Not to mention, because of its handiness in clinical settings and fascinating roles in immune and metabolism, and its direct contact with the diseased vascular wall, peripheral blood may be the most practicable tissue type for gene expression profiling. We hypothesize that analysis of gene expression profiles from peripheral blood cells would distinguish patients with hypertension from normotensives. The purpose of this novel project is to use peripheral blood cells as a surrogate tissue to distinguish patients with human essential hypertension from normotensives by gene expression profiling. Accordingly, it is hoped that understanding the genetic determinants of hypertension will contribute to improvements in prevention, diagnosis and treatment of this disorder.

\section{MATERIALS AND METHODS}

\section{Ethics Statement}

Ethical approval for the study was obtained from the Medical Faculty of China-Japan Union Hospital of Jilin University, China. All study subjects provided written informed consent for the collection of samples and subsequent analysis. This study was conducted according to the principles and guidelines expressed in the declaration of Helsinki.

\section{Study Subjects}

A total of 18 subjects $(n=18)$ were recruited in this study; 9 hypertensives and 9 normotensives. Inclusion criteria for the hypertension participants were average Systolic blood pressure (SBP) $\geq 160 \mathrm{~mm} \mathrm{Hg}$ and Diastolic blood pressure (DBP) $\geq 100 \mathrm{~mm} \mathrm{Hg}$ and absence of secondary hypertension. Inclusion criteria for the normotensives were SBP $<135 \mathrm{mmHg}$ and DBP $<85 \mathrm{~mm} \mathrm{Hg}$ and absence of family history of hypertension in first degree relatives. Exclusion criteria were diabetes, smoking, renal failure, coronary artery disease (CAD), stroke, peripheral artery disease (PAD). The variables measured were age, sex, body mass index (BMI), SBP, DBP, creatinine, triglycerides, high density lipoprotein Cholesterol (HDL-C), low density lipoprotein cholesterol (LDL-C), glucose (GLU), and high sensitivity C - reactive protein (hs-CRP).

\section{C-reactive protein (CRP) Assay}

CRP was analyzed at the clinical chemistry laboratory at the China-Japan Union Hospital, Jilin University, China. CRP was determined by using Beckman Coulter-Immunochemistry system IMMAGE 800 (Brea, California) following manufacturer's protocol. The causal mechanisms partly responsible for an increased risk of cardiovascular disease remain largely unknown. We determine whether blood pressure is associated with systemic inflammation which is a down pat risk factor for cardiovascular event.

\section{RNA processing and quantification.}

In this process, peripheral blood cells were collected from each participant. For microarray studies, we constructed 6 pools of 3 ; hypertension $(n=3)$ vs normotensive $(\mathrm{n}=3)$ and for validation through quantitative RT-PCR, hypertension $(n=9)$ vs normotensive $(n=9)$.

Total RNA was extracted using TRIzol Reagent (Invitrogen, USA) according to the method described 
by Chomczynski and Sacchi (10) and consecutively purified using the RNeasy Mini cleanup kit (QIAGEN, USA) according to manufacturer's protocol.

RNA quantity and quality were determined using a NanoDrop Technologies ND -1000 spectrophotometer (NanoDrop, Wilmington, DE 19810 USA) and an Agilent 2100 bioanalyzer (Agilent Technology, USA). Any RNA sample that degraded was excluded from the study.

\section{Microarray procedures}

Microarray study was performed at CapitalBio Corporation (Beijing, China) using Affymetrix GeneChip Human Genome U133 Plus 2.0 Array (Affymetrix, Santa Clara, CA, USA.) according to manufacturer's protocol. Total RNA $(10 \mu \mathrm{g})$ was first reverse transcribed using a T7-Oligo (dT) Promoter Primer in the first-strand cDNA synthesis reaction. Following RNase H-mediated second-strand cDNA synthesis, the double-stranded cDNA was purified and served as a template in the subsequent in vitro transcription (IVT) reaction. The IVT reaction was carried out in the presence of T7 RNA Polymerase and a biotinylated nucleotide analog/ribonucleotide mix for complementary RNA (cRNA) amplification and biotin labeling. The biotinylated cRNA targets were fragmented in $5 \times$ fragmentation Buffer provided with the GeneChip Sample Cleanup Module (Affymetrix) at $94^{\circ} \mathrm{C}$ for $35 \mathrm{~min}$. In brief, $10 \mu \mathrm{g}$ of fragmented biotin-labeled cRNA per replicate in hybridization mixture was then hybridized to Human Genome Array from Affymetrix GeneChip and incubated overnight, set to $45^{\circ} \mathrm{C}$ in Affymetrix GeneChip Hybridization Oven 640, all according to the manufacturer's instructions. After $16 \mathrm{hrs}$ of hybridization in several cycles, the mixtures were then removed, chips were washed with Non-Stringent Wash Buffer and stained with Streptavidin Phycoerythrin (SAPE) and antibody solution mix (Affymetrix) on an automated Affymetrix GeneChip Fluidics Wash Station 450. The data were collected by using Affymetrix GeneChip Scanner 3000 with workstation. Microarray images were processed and raw data were extracted with Affymetrix GeneChip Operating Software (GCOS1.4).

\section{Quantitative Real-Time RT-PCR validation of microarray gene expression patterns}

To confirm the microarray data, ten differentially expressed genes were chosen because of their different processes and perceived biological relevance to the disease. Six (CD36 molecule (thrombospondin receptor), solute carrier family4 anion exchanger member 1 (SLC4A1), neuroepithelial cell transforming 1 (NET1), sestrin 3 (SESN3), zinc finger protein 652 (ZNF652), peroxiredoxin 6 (PRDX6)) were selected for their higher expression and four (Huntingtin interacting protein 1(HIP1), folate receptor 3 (gamma) (FOLR3), endoplasmic reticulum aminopeptide 1(ERAP1), complement factor D (CFD)) for their lower expression. The primers set for the ten genes were designed using Primer3 (Table 1). Briefly, the synthesized cDNA were subjected to qPCR and amplifications performed with BioEasy SYBR Green I (Biotechs, China). Specificity of all individual amplification reactions was confirmed by melting curve analysis. Real-time expression values were calculated by using the relative standard curve method. Relative quantification method was chosen for each of the 10 genes in our study. Standard curves were generated for each mRNA by using 10 -fold serial dilutions. All reactions were based on the following recommended protocol using $0.5 \mu \mathrm{l}$ of each primer and $1 \mu \mathrm{l}$ of template per reaction.

Table I, Real-Time PCR Primers for validation of microarray data

\begin{tabular}{|c|c|c|c|c|c|}
\hline $\begin{array}{l}\text { Gene } \\
\text { Symbol }\end{array}$ & $\begin{array}{l}\text { RefSeq Tran- } \\
\text { script ID }\end{array}$ & Forward Primers & Reverse Primers & $\begin{array}{l}\text { Product size ( } \\
\text { bp) }\end{array}$ & Temp \\
\hline CD36 & NM_000072 & 5'ATGGATTAAACCCAAATGAAGA 3' & 5'CCCAGTCTCATTAAGCCAAAG 3' & 188 & $58^{\circ} \mathrm{C}$ \\
\hline SLC4A1 & NM_000342 & 5'GCTCCTATTTCCCTGGCAAG 3' & 5'CCGAGAGTTTCTGGGTGTAGGT 3' & 120 & $60^{\circ} \mathrm{C}$ \\
\hline NET1 & NM_001047160 & 5'TACAGTCATTTACCCTTCGTGG 3' & 5'TTCACCTCGGGACATTTCAT 3' & 200 & $60^{\circ} \mathrm{C}$ \\
\hline SESN3 & NM_144665 & 5'CAGGCAGCAACTTTGGGATT 3' & 5'GACGCCTCTTCATCTTCCCT 3' & 100 & $60^{\circ} \mathrm{C}$ \\
\hline ZNF652 & NM_001145365 & 5'CTGTGGAAAATCATTCAAACGC 3' & 5'TTTCGTCACAGTTCTCGCATCTA3' & 95 & $60^{\circ} \mathrm{C}$ \\
\hline PRDX6 & NM_004905 & 5'ТCTATССТСТАСССAGCTACCA 3' & 5'ТССССАТССТТССААТСАА 3' & 119 & $60^{\circ} \mathrm{C}$ \\
\hline HIP1 & NM_005338 & 5'CGGACTCAGACTGTCAGCATC 3' & 5'TGGCAGAACTTCCAGCAGAG 3' & 179 & $60^{\circ} \mathrm{C}$ \\
\hline FOLR3 & NM_000804 & 5'TGTATGGCCAGTGCAGTCCC 3' & 5'GCAGGTGGGTTCCATCTTAC 3' & 131 & $60^{\circ} \mathrm{C}$ \\
\hline ERAP1 & NM_001040458 & 5'ATGCCATTGGTGAAATCTGTG 3' & 5'CCACTCTTGGTTATCTTGCTGA 3' & 137 & $60^{\circ} \mathrm{C}$ \\
\hline CFD & NM_001928 & 5'CTGCTACAGCTGTCGGAGAAG 3' & 5'CGCGTGGTTGACTATGCC 3' & 129 & $60^{\circ} \mathrm{C}$ \\
\hline
\end{tabular}


Essentially, reactions were accomplished for each sample for 45 cycles $/ 95^{\circ} \mathrm{C} / 2 \mathrm{~min}$ denaturing step; $94^{\circ} \mathrm{C} / 15$ sec annealing/extension step and final extension step at $60^{\circ} \mathrm{C} / 40 \mathrm{sec}$ in the ABI PRISM 7500 Sequence Detection System (Applied Biosystems, USA). Data are reported as values normalized to the housekeeping gene GAPDH. One-Way ANOVA analysis was performed using SPSS (13.0) software. Significance difference between the means for each gene was evaluated using LSD or Tamhane's T2 test (significance levels at $\mathrm{P}<0.05$ ). All values are presented as means \pm SEM.

\section{Data Analysis}

Participants' demographics

Participants' demographics were recorded and analyzed using the Statistical package for Social Sciences (SPSS) for windows, version 12 (SPSS Inc., Chicago, IL, USA). All clinical parameters, including CRP were compared by student $t$ - test. The levels of CRP were compared between patients with hypertension and normotensives.

\section{Array Analysis}

The data were analyzed using SAM; Stanford University, CA, program (11). This method assigns a score to every gene on the basis of change in gene expression relative to the standard deviation of repeated measurements. To estimate the false discovery rate (FDR), SAM uses permutations of the repeated measurements, which is the percentage of genes identified by chance.

Basically, array normalization and computation of expression values of the 6 chips were performed by using DNA-Chip Analyzer (dchip) (12, 13). For annotation of the resulting genes, the gene ontology browser NetAffymetrix (http://ww.affymetrix.com) was used (14).

Similarly, the differentially expressed genes with $\geq 2$ or $\leq 0.5 \mathrm{FC}$, q-value $\leq 5 \%$ and FDRs of 5.0 or less were analyzed using $t$ test and P-value and clustered with the software package cluster 3.0.The genes were categorized more specifically by biological process ontology terms and Kyoto Encyclopedia of Genes and Genomes (KEGG) biological pathways using the software Molecule Annotation System 2.0 (http//:bioinfo.capitalbio.com/MAS/) (capitolbio, Beijing, China).

\section{Gene Ontology Analysis}

The differentially expressed genes were classified with gene ontology (GO) analysis according to their functions. In this process, we employed the $\mathrm{Z}$ scores transformation statistics to compute standardized difference score for each gene grouping (15).

\section{RESULTS}

\section{Participants' characteristics}

Table 2 displays clinical characteristics of participants. There were no significant age, gender and triglycerides differences between groups. There were significant BMI, LDL-C, HDL-C and creatinine differences between groups. The levels of CRP were significantly different between patients with hypertension and normotensives (Table 2). CRP levels were significantly higher in the hypertensive group than the normotensives.

Table 2, Clinical characteristics of participants

\begin{tabular}{llllll|}
\hline Parameter & $\begin{array}{l}\text { Hyperten- } \\
\text { sion } \\
\mathbf{n = 9}\end{array}$ & $\begin{array}{l}\text { Normoten- } \\
\text { sive } \\
\mathbf{n}=\mathbf{9}\end{array}$ & $\mathbf{t}$ & $\mathbf{P}$ \\
\hline Age, $\mathrm{y}$ & $50 \pm 4.7$ & $49 \pm 3.8$ & 0.496 & 0.626 \\
\hline Gender, M/F & $5 / 4$ & $5 / 4$ & - & $1.000^{*}$ \\
$\mathrm{BMI}, \mathrm{kg} / \mathrm{m}^{2}$ & $27 \pm 2$ & $25 \pm 1$ & 2.683 & 0.016 \\
$\mathrm{SBP}, \mathrm{mm} \mathrm{Hg}$ & $177 \pm 6$ & $120 \pm 5$ & 21.89 & $<0.00$ \\
& & & 4 & 1 \\
\hline $\mathrm{DBP}, \mathrm{mm} \mathrm{Hg}$ & $100 \pm 10$ & $79 \pm 1$ & 6.269 & $<0.00$ \\
\hline LDL-C, mmol/L & $3.5 \pm 0.9$ & $2.6 \pm 0.2$ & 2.929 & 0.010 \\
\hline HDL-C, mmol/L & $1.26 \pm 0.1$ & $1.48 \pm 0.27$ & 2.292 & 0.036 \\
\hline $\begin{array}{l}\text { Triglycerides, } \\
\text { mmol/L }\end{array}$ & $1.2 \pm 0.5$ & $1.1 \pm 0.4$ & 0.469 & 0.646 \\
\hline Glucose, mmol/L & $4.9 \pm 0.7$ & $5.1 \pm 0.2$ & 0.824 & 0.422 \\
\hline Creatinine, $\mu \mathrm{mol} / \mathrm{L}$ & $75 \pm 7$ & $81 \pm 3$ & 2.364 & 0.031 \\
\hline CRP mg/L & $3.15 \pm 0.27$ & $0.93 \pm 0.16$ & 21.22 & 0.001 \\
\hline
\end{tabular}

* Fisher's exact test. Values are shown as means \pm SDs, unless otherwise specified; " $n$ " represents the number of subjects.

\section{Microarray analysis}

Affymetrix GeneChips Human Genome U133 Plus 2.0 arrays were used to compare the population of hypertensive patients with the population of normotensives. From a total of 54681 tested probe sets, 19033 (34.80\%) were found to be present (detection P-value $<0.05)$. The data from the microarray analysis, using $\mathrm{FC} \geq 2$ or $\leq 0.5$ and q-value $\leq 5 \%$ in expression, resulted in 31 up-regulated and 18 down-regulated genes. The data discussed in this manuscript have been deposited in National center for Biotechnology information's (NCBI) Gene expression Omnibus (16) and are accessible through GEO series accession number GSE24752 (http://www.ncbi.nlm.nih.gov/ geo/query/acc.cgi?acc=GSE24752). 
Between patients with hypertension $(n=9)$ and normotensives $(n=9)$, we identified 49 differentially expressed genes: 31 up-regulated and 18 down regulated (Table 3 ). In addition to the genes validated by quantitative RT-PCR, the following genes (ABCA1, ABCG1, ACP1, HLA-DPB1 and HLA-DPB1) may be relevant to the disease, though they were not validated.

Table 3, List of statistically significantly expressed genes. Unbold and bold: up and down regulated genes, respectively.

\begin{tabular}{|c|c|c|c|c|c|}
\hline Probe Set ID & Gene Symbol & Gene Title & Score $(\mathrm{d}) *$ & Fold Change & q-value $(\%)^{\#}$ \\
\hline 242673_at & UBE3C & Ubiquitin protein ligase $\mathrm{E} 3 \mathrm{C}$ & 1.88526 & 5.88082 & 107.371 \\
\hline 1559810_at & LOC642313 & hypothetical LOC642313 & 1.694141054 & 5.667934932 & 107.3708967 \\
\hline 242197_x_at & CD36 & CD36 molecule (thrombospondin receptor) & 1.667673318 & 4.800305344 & 107.3708967 \\
\hline 223528_s_at & $\begin{array}{l}\text { LOC731602 /// } \\
\text { METT11D1 }\end{array}$ & $\begin{array}{l}\text { similar to methyltransferase } 11 \text { domain containing } \\
1 \text { isoform } 2 / / / \text { methyltransferase } 11 \text { domain } \\
\text { containing } 1\end{array}$ & 1.61409178 & 4.552732468 & 107.3708967 \\
\hline 205592_at & SLC4A1 & $\begin{array}{l}\text { solute carrier family } 4 \text {, anion exchanger, member } 1 \\
\text { (erythrocyte membrane protein band 3, Diego } \\
\text { blood group) }\end{array}$ & 1.792785531 & 3.966354548 & 107.3708967 \\
\hline 221491_x_at & $\begin{array}{l}\text { HLA-DRB1 /// } \\
\text { HLA-DRB3 /// } \\
\text { HLA-DRB4 /// } \\
\text { HLA-DRB5 /// } \\
\text { LOC100133484 } \\
/ / / / \\
\text { LOC100133661 } \\
/ / / \text { LOC731718 }\end{array}$ & $\begin{array}{l}\text { major histocompatibility complex, class II, DR beta } \\
1 / / / \text { major histocompatibility complex, class II, } \\
\text { DR beta } 3 \text { / / / major histocompatibility complex, } \\
\text { class II, DR beta } 4 \text { / / / major histocompatibility } \\
\text { complex, class II, DR beta } 5 \text { / / / similar to Major } \\
\text { histocompatibility complex, class II, DR beta } 4 \text { // / } \\
\text { similar to HLA class II histocompatibility antigen, } \\
\text { DR-W53 beta chain / / / similar to HLA class II } \\
\text { histocompatibility antigen, DRB1-7 beta chain } \\
\text { precursor (MHC class I antigen DRB1*7) (DR-7) } \\
\text { (DR7) }\end{array}$ & 4.406126791 & 3.846374234 & 0 \\
\hline 201829_at & NET1 & neuroepithelial cell transforming 1 & 1.55584681 & 3.691284279 & 107.3708967 \\
\hline 202918_s_at & MOBKL3 & $\begin{array}{l}\text { MOB1, Mps One Binder kinase activator-like } 3 \\
\text { (yeast) }\end{array}$ & 1.546033549 & 3.464079501 & 107.3708967 \\
\hline 1556988_s_at & CHD1L & $\begin{array}{l}\text { chromodomain helicase DNA binding protein } \\
\text { 1-like }\end{array}$ & 1.783724855 & 3.393248908 & 107.3708967 \\
\hline 235684_s_at & SESN3 & sestrin 3 & 1.805634293 & 3.390298507 & 107.3708967 \\
\hline 222103_at & ATF1 & activating transcription factor 1 & 1.563370465 & 3.242605034 & 107.3708967 \\
\hline 212159_x_at & $\mathrm{AP} 2 \mathrm{~A} 2$ & adaptor-related protein complex 2 , alpha 2 subunit & 1.679690033 & 2.941687252 & 107.3708967 \\
\hline 234989_at & NCRNA00084 & non-protein coding RNA 84 & 1.827840917 & 2.770613352 & 107.3708967 \\
\hline 222418_s_at & TMEM43 & transmembrane protein 43 & 3.166546718 & 2.735062637 & 107.3708967 \\
\hline 226338_at & TMEM55A & transmembrane protein $55 \mathrm{~A}$ & 1.618733749 & 2.640592116 & 107.3708967 \\
\hline 229713_at & PIP4K2A & $\begin{array}{l}\text { Phosphatidylinositol-5-phosphate 4-kinase, type } \\
\text { II, alpha }\end{array}$ & 2.5048 & 2.63686 & 107.371 \\
\hline 201863_at & FAM32A & family with sequence similarity 32 , member A & 1.55703607 & 2.583371779 & 107.3708967 \\
\hline 202838_at & FUCA1 & fucosidase, alpha-L- 1, tissue & 1.755214914 & 2.545112036 & 107.3708967 \\
\hline 205594_at & ZNF652 & zinc finger protein 652 & 1.8699841 & 2.294553891 & 107.3708967 \\
\hline 213038_at & RNF19B & ring finger protein 19B & 1.527459002 & 2.123070765 & 107.3708967 \\
\hline 204594_s_at & SMCR7L & $\begin{array}{l}\text { Smith-Magenis syndrome chromosome region, } \\
\text { candidate 7-like }\end{array}$ & 1.552814262 & 2.085802911 & 107.3708967 \\
\hline 205654_at & C4BPA & complement component 4 binding protein, alpha & 2.720863469 & 2.035574937 & 107.3708967 \\
\hline 204838_s_at & MLH3 & mutL homolog 3 (E. coli) & 1.818566911 & 1.955759304 & 107.3708967 \\
\hline 201630_s_at & ACP1 & acid phosphatase 1 , soluble & 1.598706621 & 1.944996865 & 107.3708967 \\
\hline 213872_at & C6orf62 & Chromosome 6 open reading frame 62 & 1.803756915 & 1.927246979 & 107.3708967 \\
\hline 212473_s_at & MICAL2 & $\begin{array}{l}\text { microtubule associated monoxygenase, calponin } \\
\text { and LIM domain containing } 2\end{array}$ & 2.117996134 & 1.812521326 & 107.3708967 \\
\hline 200844_s_at & PRDX6 & peroxiredoxin 6 & 1.504701696 & 1.730976848 & 107.3708967 \\
\hline 214525_x_at & MLH3 & mutL homolog 3 (E. coli) & 1.632503504 & 1.697662053 & 107.3708967 \\
\hline 218676_s_at & PCTP & phosphatidylcholine transfer protein & 1.666238817 & 1.622913043 & 107.3708967 \\
\hline 217902_s_at & HERC2 & hect domain and RLD 2 & 1.807675847 & 1.620439623 & 107.3708967 \\
\hline 222730_s_at & ZDHHC2 & zinc finger, DHHC-type containing 2 & 1.912364228 & 1.525083989 & 107.3708967 \\
\hline 209906_at & C3AR1 & complement component 3 a receptor 1 & -1.678515094 & 0.661637838 & 107.40645 \\
\hline 201137_s_at & HLA-DPB1 & $\begin{array}{l}\text { major histocompatibility complex, class II, DP } \\
\text { beta } 1\end{array}$ & -1.526092306 & 0.650013114 & 107.40645 \\
\hline 244228_at & RABGAP1 & RABGTPase activating protein 1 & -1.58675 & 0.63685 & 107.406 \\
\hline 203504_s_at & ABCA1 & $\begin{array}{l}\text { ATP-binding cassette, sub-family A (ABC1), } \\
\text { member } 1\end{array}$ & -1.79577537 & 0.620203666 & 107.40645 \\
\hline
\end{tabular}




\begin{tabular}{|c|c|c|c|c|c|}
\hline 204861_s_at & NAIP & NLR family, apoptosis inhibitory protein & -1.628234316 & 0.616196823 & 107.40645 \\
\hline 236495_at & NAMPT & Nicotinamide phosphoribosyltransferase & -1.56998 & 0.61063 & 107.406 \\
\hline 204860_s_at & NAIP & NLR family, apoptosis inhibitory protein & -1.840825605 & 0.608945029 & 107.40645 \\
\hline 1557050_at & hoxa2 & homeobox A2 & -1.75782 & 0.5996 & 107.406 \\
\hline 204567_s_at & ABCG1 & $\begin{array}{l}\text { ATP-binding cassette, sub-family G (WHITE), } \\
\text { member } 1\end{array}$ & -1.766317544 & 0.575507906 & 107.40645 \\
\hline 226364_at & HIP1 & Huntingtin interacting protein 1 & -1.561453912 & 0.493465276 & 107.40645 \\
\hline 206371_at & FOLR3 & folate receptor 3 (gamma) & -1.698220762 & 0.469677494 & 107.40645 \\
\hline 214012_at & ERAP1 & endoplasmic reticulum aminopeptidase 1 & -1.84135062 & 0.461242008 & 107.40645 \\
\hline 228642_at & hoax & homeobox A2 & -1.84056 & 0.44361 & 107.406 \\
\hline 214012_at & $\begin{array}{l}\text { HLA-DQA1 /// } \\
\text { HLA-DQA2 }\end{array}$ & $\begin{array}{l}\text { major histocompatibility complex, class II, DQ } \\
\text { alpha } 1 / / / \text { major histocompatibility complex, } \\
\text { class II, DQ alpha } 2\end{array}$ & -1.61069578 & 0.441730691 & 107.40645 \\
\hline 212671_s_at & TCL1A & T-cell leukemia/lymphoma 1A & -1.813983234 & 0.421260975 & 107.40645 \\
\hline 226736_at & CHURC1 & Churchill domain containing 1 & -1.53264385 & 0.416866185 & 107.40645 \\
\hline 1558048_at & & & -1.982 & 0.41038 & 107.408 \\
\hline 205382_at & CFD & Complement factor D (adipsin) & -1.817904316 & 0.378262489 & 107.40645 \\
\hline
\end{tabular}

* The student's $t$-statistic value; \#The lowest False Discovery Rate at which the gene is called significant

\section{Validation of microarray results by real-time quantitative RT- PCR analysis}

Ten genes were selected for further analysis and validation of microarray results using quantitative RT-PCR. These include CD36, SLC4A1, NET1, SESN3, ZNF652 and PRDX6 (up-regulated) and HIP1, FOLR3, ERAP1 and CFD (down-regulated). The values were presented as means \pm SEM (Table 2). SPSS analysis shows that the expression of CD36 (p., 0.000), SLC4A1 (p., 5.091e-005), NET1 (p., 3.56e-008), SESN3 (p., 1.181e-005), ZNF652 (p., 0.000), PRDX6 (p., 0.000) are up-regulated and HIP1 (p., 0.000), FOLR3 (p., 0.000), ERAP1 (p., 0.004) and CFD (p., 0.006) are down-regulated (Fig. 1). The real-time quantitative
RT-PCR results were consistent with that of microarray.

\section{Gene Ontology (GO) Analysis}

The most statistically significantly overrepresented GO terms are displayed in Table (4) and several of them are related to immune/inflammation responses. Interestingly, "Major histocompatibility complex class II receptor activity (MHC), Immune response and MHC class II protein complex" were identified as the most statistically significantly GO terms. These GO terms may play important role in the patho-biology of essential hypertension.

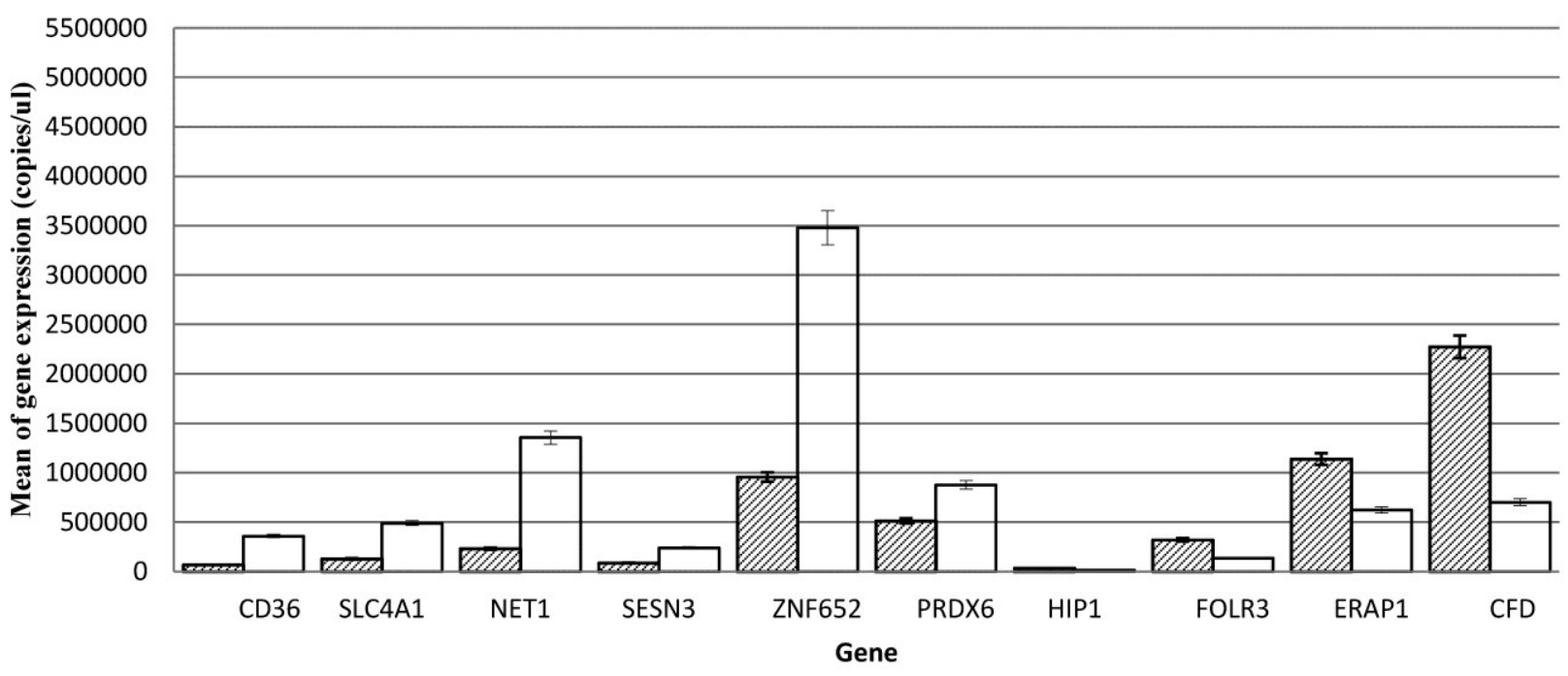

Figure I, Quantitative PCR measurements of gene expression of CD36, SLC4AI, NETI, SESN3, ZNF652, PRDX6, HIPI, FOLR3, ERAPI, and CFD from peripheral blood cells samples of patients with hypertension and normotensives. Significant levels at $(p<0.05)$. 


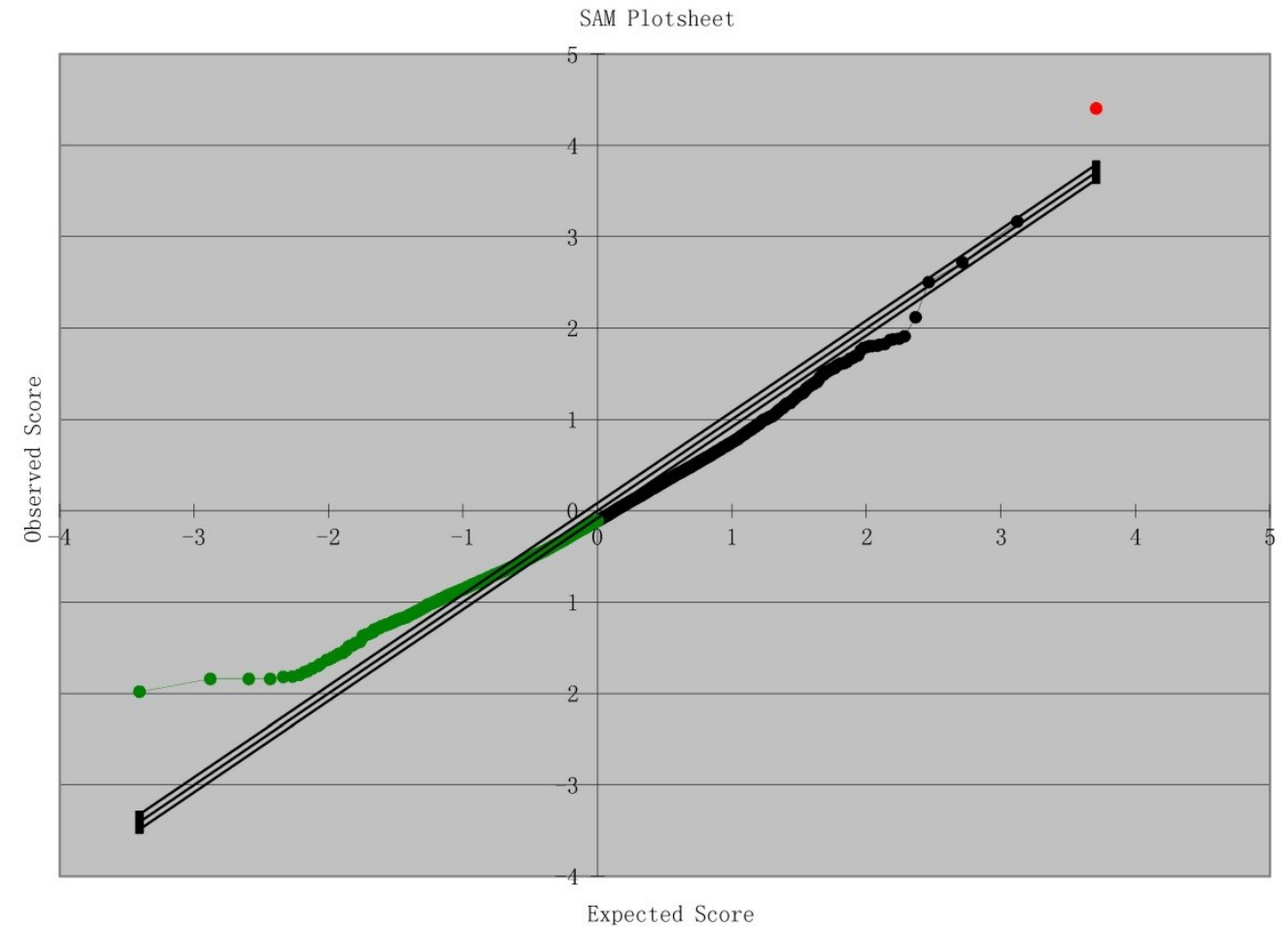

Figure 2, Results of Microarray Analysis: SAM Plot. Significant: 9060; Median number of false positives: 9731.02; False discovery rate (\%): I07.4I; Tail strength (\%): -4I.I; se (\%): 29.2

Table 4, statistically significantly overrepresented GO terms

\begin{tabular}{|c|c|c|c|c|}
\hline GO & GO TERM & $\begin{array}{l}\text { Number of Genes in- } \\
\text { volved }\end{array}$ & P-Valu & $\begin{array}{l}\text { Q-Valu } \\
\text { e }\end{array}$ \\
\hline \multirow{10}{*}{$\begin{array}{l}\text { Molecular } \\
\text { Function }\end{array}$} & MHC Class II receptor activity & 2 & 0.0 & 0.0 \\
\hline & Cholesterol transporter activity & 2 & $2.3 \mathrm{E}-5$ & $4.1 \mathrm{E}-5$ \\
\hline & Zinc ion binding & 9 & $5.4 \mathrm{E}-5$ & $5.8 \mathrm{E}-5$ \\
\hline & Cholesterol binding & 2 & $6.1 \mathrm{E}-5$ & $6.1 \mathrm{E}-5$ \\
\hline & Phospholipid transporter activity & 2 & 2.05E-4 & $1.87 \mathrm{E}-4$ \\
\hline & Toxin transporter activity & 1 & 7.16E-4 & $4.4 \mathrm{E}-4$ \\
\hline & Complement component $\mathrm{C} 3 \mathrm{a}$ receptor activity & 1 & 7.16E-4 & $4.4 \mathrm{E}-4$ \\
\hline & Apolipoprotein A-1 receptor activity & 1 & 7.16E-4 & $4.4 \mathrm{E}-4$ \\
\hline & Glycoprotein Transporter activity & 1 & 7.16E-4 & $4.4 \mathrm{E}-4$ \\
\hline & C3a anaphylatoxin receptor activity & 1 & 7.16E-4 & $4.4 \mathrm{E}-4$ \\
\hline \multirow{11}{*}{$\begin{array}{l}\text { Biological } \\
\text { process }\end{array}$} & Immune response & 5 & 0.0 & 0.0 \\
\hline & $\begin{array}{l}\text { Antigen processing \& presentation of peptide or polysaccharide antigen via MHC } \\
\text { class II }\end{array}$ & 3 & 0.0 & 0.0 \\
\hline & Phospholipid homeostasis & 2 & $3.0 \mathrm{E}-6$ & $7.0 \mathrm{E}-6$ \\
\hline & Intracellular cholesterol transport & 2 & $5.0 \mathrm{E}-6$ & $1.0 \mathrm{E}-5$ \\
\hline & Reverse cholesterol transport & 2 & $1.8 \mathrm{E}-5$ & $3.5 \mathrm{E}-5$ \\
\hline & Phospholipid efflux & 2 & $2.3 \mathrm{E}-5$ & $4.1 \mathrm{E}-5$ \\
\hline & Cholesterol efflux & 2 & $4.0 \mathrm{E}-5$ & $5.4 \mathrm{E}-5$ \\
\hline & Positive regulation of lipid metabolism & 2 & $1.52 \mathrm{E}-4$ & 1.47E-4 \\
\hline & Foam cell differentiation & 2 & 1.77E-4 & $1.68 \mathrm{E}-4$ \\
\hline & Cholesterol metabolism & 2 & 2.05E-1 & 1.87E-4 \\
\hline & Blood Pressure regulation & 1 & 0.0537 & $\begin{array}{l}0.01366 \\
6\end{array}$ \\
\hline \multirow{2}{*}{$\begin{array}{l}\text { Cellular } \\
\text { Compo- }\end{array}$} & MHC class II protein complex & 3 & 0.0 & 0.0 \\
\hline & Integral to plasma membrane & 7 & $2.6 \mathrm{E}-5$ & $4.1 \mathrm{E}-5$ \\
\hline
\end{tabular}




\begin{tabular}{lllll}
\hline nent & Integral to membrane & 9 & $4.5 \mathrm{E}-5$ & $5.4 \mathrm{E}-5$ \\
Membrane & 13 & $2.7 \mathrm{E}-4$ & $2.22 \mathrm{E}-4$ \\
Lysosome & 3 & $3.16 \mathrm{E}-4$ & $2.37 \mathrm{E}-4$ \\
Golgi apparatus & 5 & $3.65 \mathrm{E}-4$ & $2.64 \mathrm{E}-4$ \\
Nucleus & 13 & $4.01 \mathrm{E}-4$ & $2.69 \mathrm{E}-4$ \\
Membrane Fraction & 4 & 0.00117 & $6.87 \mathrm{E}-4$ \\
Plasma membrane & 7 & 0.00139 & $7.63 \mathrm{E}-4$ \\
& & 2 & 0.00357 \\
Phagocytic Vesicle & 1 & 0.00161 \\
\hline
\end{tabular}

GO analysis was applied to differentially expressed genes. The number of transcripts calculated P-values and Q-values are shown.

\section{KEGG biological pathway}

Biological pathway analysis incriminated related pathways which were significantly modulated across patients with hypertension vs. normotensives. Several immune-related pathways were significantly overrepresented which provides evidence that immune/inflammatory responses may be associated with hypertension, including Cell adhesions molecules, Systemic lupus erythematosus, Hematopoietic cell lineage, Asthma, Graft-versus-host disease, Allograft rejection, Autoimmune thyroid disease, Antigen processing and presentation, Type 1 diabetes mellitus, and Complement and coagulation cascades.

\section{DISCUSSION}

In this study, we have successfully used gene expression profiling in peripheral blood cells to identify differences in transcription profile of human essential hypertension compared with normotensives. We chose to profile the blood in hypertensive patients because it is a sample that reflects ongoing pathologic processes such as inflammation and structural abnormalities. And therefore, inflammatory-related genes were expected to be modulated in our experiment.

In regards to limitation of the study, our sample size is relatively small due to the significant costs involved in microarray experiment. In addition, the microarray technology has decreased sensitivity to detect low-abundance genes. In spite of these limitations, our study reveals novel features of human essential hypertension.

Firstly, we investigated differences in transcription profile of human essential hypertension compared with normotensives. We identified 31 up-regulated genes and 18 down-regulated genes with FC of $\geq 2$ or $\leq 0.5$ and q-value $\leq 5 \%$ in expression. These genes and GO categories identified as being differentially expressed in essential hypertension versus normotensives may be of significant biological interest. To the best of our knowledge, this is the first time several of these genes become candidates for further investigation in their role in hypertension. Through quantitative RT-PCR, we confirmed the expression of 10 (CD36, SLC4A1, NET1, SESN3, ZNF652, PRDX6, HIP1, FOLR3, ERAP1 and CFD) out of the 49 differentially expressed genes. ERAP1 was previously shown to be an important player in hypertension. ERAP1 functions in several biological networks such as regulation of blood pressure, angiogenesis and presentation of antigens to MHC class I $(17,18,19)$ as well as immune and inflammatory responses (20). A possible role of ERAP1 in blood pressure regulation may be through its inactivation of angiotensin II and/or generation of bradykinin (17, 20).

CD36 is a scavenger receptor expressed in macrophages, endothelial cells, denditic cells and in tissues such as muscle, heart and fat $(21,22)$. Increasing evidence underscores the potential role of CD36 in the pathogenesis of hypertension. One possibility is through the regulatory effect of endothelin-1 on CD36 which might alter the properties of vascular smooth muscle cells (VSMCs), thus leading to the development of hypertension and atherosclerosis (23). Pravenec and Kurtz demonstrated that deficiency in renal expression of CD36 could promote increase in blood pressure (24).

The human anion exchanger 1 (AEI or SLC4A1) gene is a 911-residue glycoprotein that encodes protein in erythrocytes and basolateral surface of the alpha-intercalated cell in the collecting duct of the kidney $(25,26)$. Intriguingly, this gene is a possible candidate for essential hypertension as its encoded protein does not only function as chloride/bicarbonate exchanger but also in pathways that regulate blood pressure (27). Besides, Kokubo et al. (28) reported association of SLC4A1 with both blood pressure variation and hypertension. Association between ZNF652 gene and systolic or diastolic blood pressure and hypertension has been reported elsewhere $(29,30)$, however, its mechanistic role(s) were not defined. 
NET1 and SESN3 have been suggested to be associated with genotoxic stress and /or oxidative stress $(31,32,33)$. Short while, oxidative stress has been implicated in the pathophysiology of hypertension (34,
$35,36)$. It is believed that an imbalance in superoxide and nitric oxide generation may lead to vasodilation and subsequently hypertension (Figure 3).

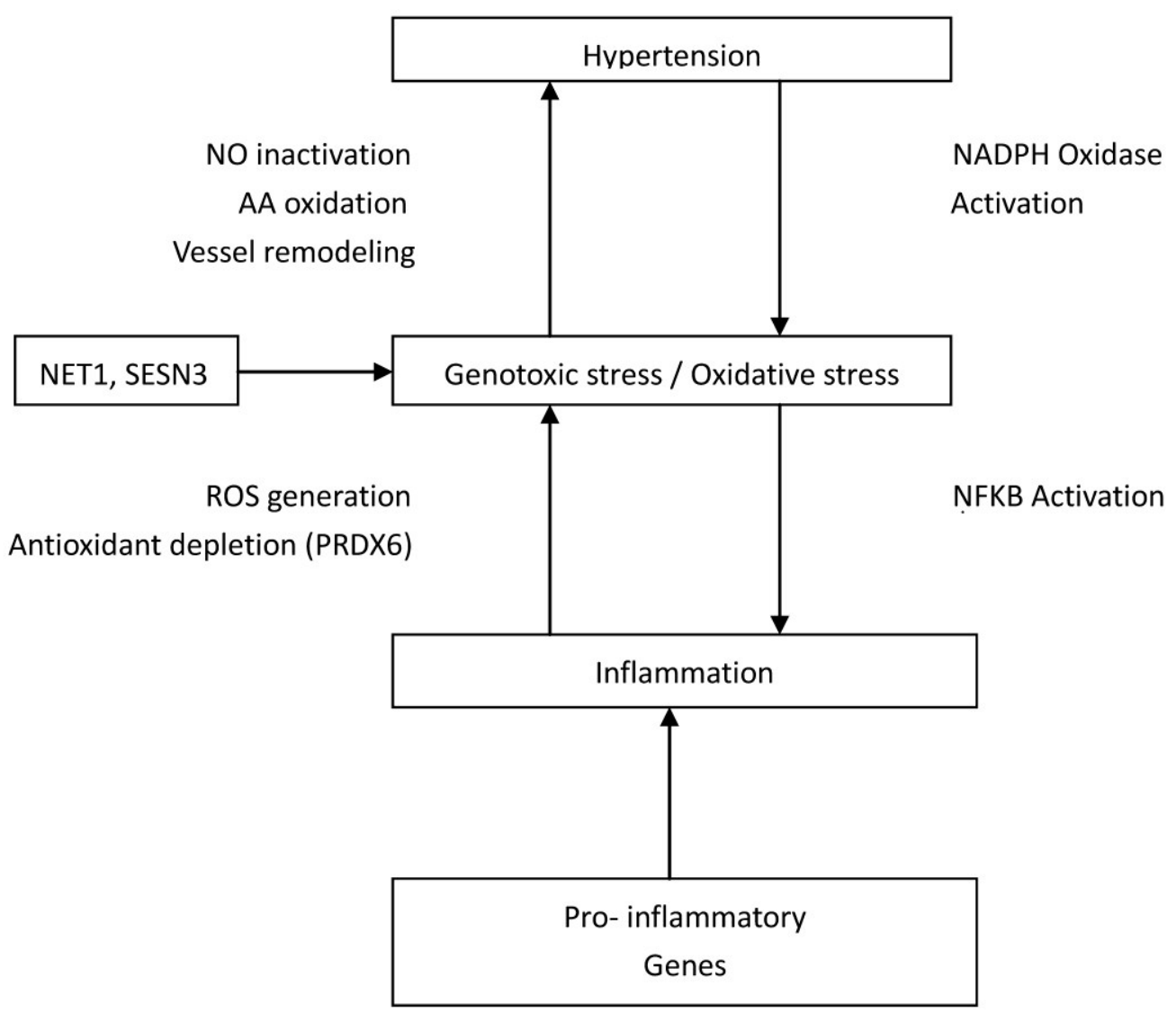

Figure 3, Interconnections between Genes and their relationship to Hypertension. NO, indicates nitric oxide; AA, arachidonic acid; ROS, reactive oxygen species; NFKB, nuclear factor-kappa B; NADPH, nicotinamide adenine dinucleotide phosphate.

An increase in production of ROS enhanced depletion of antioxidant (PRDX6) which may cause oxidant stress thereby leading to hypertension (37). The PRDX6 gene has also been implicated as a candidate gene for atherosclerosis (38). Seemingly, it has been proposed that HIP1 mutations lead to abnormal hematopoiesis (39) and Park\& Zambidis (40) have suggested a role of rennin-angiotensin system (RAS) in hematopoiesis. The critical physiological roles of RAS in blood pressure regulation could be a possible link between HIP1 and hypertension. FOLR3 has a high affinity for folic acid and folic acid has been reported to reduce serum homocysteine levels as well as systolic and diastolic blood pressure, in addition to improving the integrity of vascular endothelium (41, 42). In the study of Gomez-Ambrosi et al. (43), FOLR3 was differentially expressed in human omental adi- pose tissue of obese individuals. The differential expression of this gene in both obese and hypertensive individuals demonstrate further evidence for a consistent link between adiposity and hypertension. CFD is an immune/inflammatory-related gene involved in complement and coagulation cascades. Its role in hypertension biology could be through its association with pathological angiogenesis. The excessive, insufficient or abnormal angiogenesis has been implicated in the pathogenesis of several cardiovascular diseases, including hypertension $(44,45)$.

Other modulated genes in our experiment, though were not validated by quantitative RT-PCR but maybe associated with and/or play role in hypertension include ABCA1, ABCG1, ACP1, HLA-DPB1, and HLA-DRB3. ATP binding cassette transporters (ABCA1, ABCG1) are important cellular 
cholesterol transporters/receptor in regulating cholesterol efflux. The implication of these genes as candidate genes for hypertension has been substantially catalogued $(46,47)$. ABCA1 mutations may increase the risks of both cardiovascular diseases and type 2 diabetes by reducing plasma high density lipoprotein (48). However, their mechanistic roles in the pathogenesis of essential hypertension remain irresolute.

Acid phosphate 1, soluble (ACP1) has been found to be related to CAD and was differentially expressed in human omental adipose tissue of obese individuals $(49,43)$. The up-regulation of ACP1 in our study reinforces the notion that hypertension is indeed a disorder of cardiovascular endocrinology and metabolism. Furthermore, our study shows that HLA-DPB1 and HLA-DRB3 may play important role in hypertension. Association of these genes with cardiovascular, metabolic and immune diseases has been reported $(50,51,52)$.

Table 4 lists GO categories and specific number of genes with their respective categories that were differentially expressed in peripheral blood samples of patients with hypertension compared with normotensives. The "MHC class II receptor activity" and "immune response" are intriguing and may be associated with human essential hypertension.

Biological pathway analysis identified several related pathways. The overexpression of these immune-related pathways may show that hypertension may be associated with immune/inflammatory responses.

Secondly, we analyzed the levels of CRP in patients with hypertension by measuring CRP levels in participants included in the study. The levels of inflammatory marker, CRP were elevated in hypertensive patients than the normotensives (Table 2). This finding which shows that inflammation is associated with hypertension is in concordant with many other studies $(53,54)$. Of note, inflammations in individuals with hypertension have been clearly established, however, its contributory roles to the etiology of hypertension is probably secondary to hypertension. Endothelial dysfunction and angiotensin type 1 might be hypercritical in the development of hypertension. Recently, several studies have focused on endothelium and angiotensin molecular networks in the pathogenesis of hypertension, proposing that inflammation is associated with endothelium damage and the RAS. However, these questions, "Does inflammation lead to hypertension or does hypertension induce inflammation?" necessitate further investigation.

Taken together, this study has demonstrated gene expression changes in peripheral blood cells that accurately distinguish patients with human essential hypertension from normotensives. We have also identified a number of novel genes, GO categories and biological pathways that may be associated with the pathobiology of human essential hypertension. Moreover, our study has shown that inflammation is associated with hypertension. It is anticipated that understanding the genetic background of essential hypertension will provide accurate and efficient strategies for prevention, diagnosis and control.

\section{ACKNOWLEDGEMENTS}

Acknowledgement to Professor Zhihui Zhao, Dean College of Animal Science and Veterinary medicine, Jilin University for using his lab and Dr. Lihong Qin for her technical support. Our special thanks go to the three anonymous referees for comments that immensely improved the manuscript. This work was supported by a 30 million grant from the National Science Foundation of Growth Hormone, Heart Failure, and Inhibition of cardiomyocyte apoptosis and protective effect of endothelial cell, 20081-201012.Item \# 30770883 P.R. China.

\section{CONFLICT OF INTEREST}

The authors declared no conflict of interest.

\section{REFERENCES}

1. Chockalingm A, Campbell NR, Fodor JG. Worldwide epidemic of hypertension. Can J Cardio. 2006; 22:553-555.

2. Tomson J, Lip GYH. Blood Pressure demographic: nature or nurture...... genes or environment? BMC Med. 2005; 3:3.

3. WHO. World Health Report 2002: Reducing Risks, Promoting Healthy life. Geneva: World Health Organization. 2002.

4. Heller RA et al. Discovery and analysis of inflammatory disease related genes using cDNA microarrays. Proc Nat Acad Sci. 1997; 94: 2150- 2155.

5. Lockhart DJ, Dong H, Byrne MC, Follettie MT, Gallo MV, Chee MS,Mittmann M, Wang C, Kobayashi M, Horton H, Brown EL. Expression monitoring by hybridization to high-density oligonucleotide arrays. Nat Biotechnol.1996; 14:1675-80.

6. Tzouvelekis A, Patlakas G, Bouros D. Application of Microarray Technology in pulmonary diseases. Respir Res. 2004;5:26.

7. King HC, Sinha AA. Gene expression profile analysis by DNA microarrays: promise and pitfalls. JAMA. 2001; 286:2280-2288.

8. LI JJ. Inflammation in hypertension: primary evidence. Chin Med J 2006; 119: 1215-21.

9. Pauleto P, Rattazzi M. Inflammation and Hypertension: the search for a link. Nephrol Dial Transplant.2006; 21: 850-53.

10. Chomczynski P and Sacchi N. Single step method of RNA isolation by acid guanidinium thiocyanate-phenol-chloroform extraction. Anal Biochem. 1987; 162:156-159.

11. Tusher VG, Tibshirani R, Chu G. Significance analysis of microarrays applied to the ionizing radiation response. Proc Natl Acad Sci USA.2001; 98:5116-5121.

12. Li C, Hung Wong W. Model-based analysis of oligonucleotide arrays: model validation, design issues and standard error application. Genome Biol. 2001; 2(8):RESEARCH0032. 
13. Li C, Wong WH. Model-based analysis of oligonucleotide arrays: expression index computation and outlier detection. Proc Natl Acad Sci. 2001; 98:31-6.

14. Liu G, Loraine AE, Shigeta R, Cline M, Cheng J, Valmeekam V, Sun S, Kulp D, Siani-Rose MA. NetAffx: Affymetrix probe sets and annotations. Nucleic Acids Res. 2003; 31:82-6.

15. Cheadle C, Vawter MP, Freed WJ, Becker KG. Analysis of microarray data using Z score transformation. J Mol Diagn.2003; 5:73-81.

16. Edgar R, Domrachev M, Lash AE. Gene expression Omnibus: NCBI gene expression and hybridization array data. Nucleic Acids Res.2002; 30:2077-2210.

17. Yamamoto N, Nakayama J, Yamakawa-Kobayashi K, Hamaguchi $\mathrm{H}$, Miyazaki R, Arinami T. Identification of 33 polymorphisms in the adipocyte-derived leucine aminopeptidase (ALAP) gene and possible association with hypertension. Hum mutat. 2002; 19:251-257.

18. Sato Y. Role of aminopeptidase in angiogenesis. Biol Pharm Bull. 2004; 27:772-776.

19. Chang SC, Momburg F, Bhutani N, Goldberg A. The ER aminopeptidase, ERAP1, trims precursors to lengths of MHC class Peptidase by a "Molecular ruler" mechanism. PNAS. 2005; 102:17107-17112.

20. Hatorri A, Kiyayani K, Matsumoto H, Miyazawa S, Rogi T, Tsuruoka N, Mizutani S, Natori Y, Tsujimoto M. Characterization of recombinant human adipocyte-derived leucine aminopeptidase expressed in Chinese hamster ovary cell. J Biochem. 2000; 28: 755-762.

21. Nicholson AC, Han J, Febbraio M, Silversterin RL, Hajjar DP. Role of CD36, the macrophage class B scavenger receptor, in atherosclerosis. Ann. N. Y. Acad. Sci. 2001; 947:224-8.

22. Febbraio M, Silverstein RL. CD36: Implications in Cardiovascular Diseases. Int. J Biochem Cell Biol. 2007; 11:2012-2030.

23. Kwok CF, Juan CC, HO LT. Endothelin-1 decreases CD36 protein expression in vascular smooth muscle cells. American physiology Society 2006;10:1152.

24. Pravenec M, Kurtz Tw. Molecular genetics of experimental hypertension and metabolic syndrome: From Gene pathway to New Therapies. Hypertension.2007; 49:941-952.

25. Popov M, Li J, Reithmeier RAF. Transmembrane folding of the human erythrocyte anion exchanger (AEI, Band 3) determined by scanning and insertional N-glycosylation mutagenesis. Biochem J. 1999; 339:269-279.

26. Tanner MJA, Martin PG, High S. The complete amino acid sequence of the human erythrocyte membrane anion-transport protein determined from the cDNA sequence. Biochem J. 1998; 256:703-712.

27. Knight J, Munroe PB, Pembroke JC, Caulfield MJ. Review: Human chromosome 17 in Essential hypertension. Annals of Hum Genetics. 2003; 67:193-206.

28. Kokubo Y, Tomoike H, Tanaka C, et al. Association of sixty-one non-synonymous polymorphisms in fifty-one hypertension candidate genes with blood pressure variation and hypertension. Hyppertesion Research. 2006; 29:611-619.

29. Newton-Cheh C, Johnson T, Gateva V et al. Genome wide association study identifies eight loci associated with blood pressure. Nat Genet. 2009; 41: 666-76.

30. Niu W, Zhang Y, Ji K, Gu M, Gao P, Zhu D. Confirmation of top polymorphisms in Hypertension genome wide association study among Han Chinese. Clin Chim Acta. 2010;411(19-20):1491-5

31. Guerra L, Carr Hs, Richter Dahlfars A, Masucci MG, Theletam M, Frost JA, Fristan T. A bacterial cytotoxin identifies the RhoA exchanger factor Net1 as a key effector in the response to DNA damage. PLos One 2008;3(5):e2254.
32. Kopnin, P.B., et al. Repression of sestrin family genes contributes to oncogenic Ras-induced reactive oxygen species upregulation and genetic instability. Cancer Res.2007; 67: 4671-4678.

33. Levine AJ, Hu W, Feng Z. The P53 pathway: what questions remain to be explaned? Cell death and differentiation.2006; 13:1027-1036.

34. De champlain J, Wu R, Girouard H, Karas M, Midaoui A, Laplante MA, Wu L. Oxidative stress in hypertension. Clin Exp Hypertens. 2004; 26: 593-601.

35. Grossman E. Does increased oxidative stress cause hypertension? Diabetes care.2008; 31:185-189.

36. Ceriello A. Possible role of oxidative stress in the pathogenesis of hypertension. Diabetes Care. 2008; 31: s181-s184.

37. Wassmann S, Wassmann K, Nickenig G: Modulation of oxidant and antioxidant enzyme expression and function in vascular cells. Hypertension.2004; 44:381-386.

38. Wang X, Phelan AS, Petros C, Taylor EF, Ledinski G, Jürgens G, Forsman-Semb K, Paigen B. Peroxiredoxin 6 deficiency and atherosclerosis susceptibility in mice. Significance of genetic background for assessing atherosclerosis. Atherosclerosis.2004; 177:61-70.

39. Oravecz-wilson KL, Kiel MJ, Li L, Rao DS, Saint-Dic D, Kumar PD, Provot MM, Hankenson KD, Reddy VN, Lieberman AP, Morrison SJ, Ross TS. Huntingtin Interacting protein 1 mutations lead to abnormal hematopoiesis, spinal defects and cataracts. Human Molecular Genetics.2004; 13:851-867.

40. Park TS, Zambidis ET. A role for the rennin-angiotension system in hematopoiesis. Haematologica. 2009 Jun;94(6):745-7.

41. Papandreou D, Malindretos P, Arvanitidou M, Makedou A, Rousso I. Homocysteine lowering with folic acid supplements in children: effects on blood pressure. International journal of food sciences and nutrition. 2010; 61:11-7.

42. Palomba S, Falbo A, Giallauria F, Russo T, Tolino A, Zullo F, Colao A, Orio F. Effects of metformin with or without supplementation with folate on homocysteine levels and vascular endothelium of women with polycystic ovary syndrome. Diabetes Care. 2010;33: 246-51.

43. Gomez-Ambrosi J, Catalan V, Diez-Caballero A, Martinez-Cruz LA, Gil MJ, Garcia-Foncillas J, Cien Fuegos JA, Salvador J, Mato JM, Fruhbeck G. Gene expression profile of omental adipose tissue in human obesity. FASEB J. 2004 Jan;18(1):215-7.

44. Boudier HA. Arteriolar and capillary remodeling in hypertension. Drugs.1999; 58: 37-40.

45. Le Noble FA, Stassen FR, Hacking WJ, struilker Boudier HA. Angiogenesis and Hypertension. J. Hypertens.1998;16:1563-72.

46. Yamada et al. Association of polymorphisms of ABCA1 and ROS1 with hypertension in Japanese candidates. Int. journal of molecular medicine. 2008; 21:83-89.

47. Xu M, Zhou H, Gu Q, Li C. The expression of ATP-binding cassette transporters in hypertensive patients. Hypertension Research. 2009; 32:455-4611.

48. Tang C, Oram JF. The cell cholesterol exporter ABCA1 as a protector from cardiovascular diseases and diabetes. Biochim Biophys Acta. 2009; 19: 563-72.

49. Banci M, Saccucci P, Annibale FD, Dofcaci A, Triofera G, Magrini A, Bottini N, Bottini FG. ACP1 genetic polymorphism and Coronary Artery Disease: An Association study. Cardiology. 2009; 113:236-242.

50. Shen GQ, Moravec C, Ellis S, et al. Identification of new genes differentially expressed in coronary disease by expression profiling. Physiol. Genomics. 2003; 15:65-74.

51. Richeldi L, Sorrentino R, Saltini C. HLA-DPB1 glutamate 69: a genetic marker of beryllium disease. Science 1993;262: 197-198.

52. Terai M, Kohno Y, Namba M, Umemiya T, Niwa K, Nakajima $\mathrm{H}$, Mikata A. Class II major histocompatibility antigen expression on coronary arterial endothelium in a patient with Kawasaki disease. Hum Pathol 1990;21: 231-234. 
53. Sesso HD, Buring JE, Rifai N, Blake GJ, Gaziano JM, Ridker PM. $\mathrm{C}$-reactive protein and the risk of developing hypertension. JAMA. 2003; 290: 2945-51.

54. Boos CJ, Lip GYH. Is hypertension an inflammatory process? Current Pharmaceutical Design.2006; 12: 1623-1635.

\section{AUTHOR BIOGRAPHY}

Professor Ping Yang currently heads the Department of cardiovascular medicine, China- Japan Union Hospital, Jilin University, China. Prof. Yang is a seasoned physician and deeply perceptive research scientist with 15 years of research experience in cardiovascular medicine. She also heads the Institute of cardiovascular research, Jilin Province. Prof. Yang has a distinctive interest in the molecular mechanism of coronary heart disease (CHD) and diabetes mellitus (DM). In this light, she has published several papers with high impact factor journals in this area. She is an Editor and Reviewer of several journals including "Chinese Journal of Gerontology, Journal of Experimental Diagnostic" and "Evidence-based Cardiovascular Diseases".

Melvin T. Korkor is a candidate for Doctor of philosophy (Ph.D) in Medicine at Jilin University, P.R.China. His area of research interest is cardiovascular genetics and nitric oxide and reactive oxygen species. 